\title{
Lithium in a metal-poor external galaxy: $\omega$ Centauri
}

\author{
P. Bonifacio ${ }^{1,2}$, L. Monaco ${ }^{3,4}$. L. Sbordone ${ }^{5}$, S. Villanova ${ }^{3}$, and \\ E. Pancino ${ }^{6}$ \\ ${ }^{1}$ GEPI, Observatoire de Paris, CNRS, Université Paris Diderot; \\ Place Jules Janssen, 92190 Meudon, France \\ email: Piercarlo.Bonifacio@obspm.fr \\ ${ }^{2}$ Istituto Nazionale di Astrofisica, Osservatorio Astronomico di Trieste, \\ Via Tiepolo 11, I-34143 Trieste, Italy \\ ${ }^{3}$ Universidad de Concepción, Casilla 160-C, Concepción, Chile \\ ${ }^{4}$ European Southern Observatory, Casilla 19001, Santiago, Chile \\ ${ }^{5}$ Max Planck Institut for Astrophysics Karl-Schwarzschild-Str. 185741 Garching, Germany \\ ${ }^{6}$ Istituto Nazionale di Astrofisica, Osservatorio Astronomico di Bologna, \\ Via Ranzani 1, 40127, Bologna, Italy
}

\begin{abstract}
Centauri is a massive stellar system which is currently going through the Galactic Halo. Its compact aspect and spheroidal shape have for a long time led to it being classified as a Globular Cluster. However the fact that its stars cover a wide metallicity range $(-0.6<[\mathrm{Fe} / \mathrm{H}]<$ -2.1 ), points to this object as an external galaxy, satellite of the Milky Way. Lithium among warm metal-poor stars shows a roughly constant abundance, the "Spite Plateau". This has been interpreted as evidence for a primordial origin of the lithium nucleus, at the time of nucleosynthesis. After the physical conditions under which nucleosynthesis occurred, have been constrained by the observations of the fluctuations of the Cosmic Microwave Background, we are facing a "cosmological lithium problem", namely the primordial lithium was a factor of three to four higher than what observed in the Spite plateau. Several avenues may be taken to solve this conundrum, either relying on fundamental physics or on stellar physics, however the realm of possibilities may be considerably narrowed by observing stellar populations in different galaxies, which have experienced different evolutionary histories. Some of the proposed "solutions" may be clearly ruled out, depending on the observation of lithium in the metal-poor populations of external galaxies. $\omega$ Centauri is the only external galaxy amenable to such an investigation in the era of $8 \mathrm{~m}$ telescopes. We have pushed to its limits FLAMES at the ESO $8.2 \mathrm{~m}$ telescope to obtain high resolution spectra of the Li I doublet in 91 Turn-Off and Sub-Giant stars at V 18 in $\omega$ Centauri. We present our preliminary results on this data which suggest that the Li content in $\omega$ Centauri warm stars is comparable to that observed in Galactic Halo field stars of similar metallicities and temperatures. This may effectively rule out a whole class of models which invoke a severe Li depletion through processing of material in an early generation of massive stars.
\end{abstract}

Keywords. Nuclear reactions, nucleosynthesis, abundances - stars: abundances, Population II Galaxy: globular clusters: individual ( $\omega$ Cen) - galaxies: abundances, Local Group - cosmology: observations

\section{Introduction}

The Spite plateau is the constant Li abundance, observed in warm metal-poor stars of different effective temperature and metallicity. This remarkable feature in the abundance pattern of metal-poor stars was discovered by Monique and François Spite in 1982 
(1982a,1982b) and was immediately interpreted as a signature of Big Bang Nucleosynthesis (BBN) and a mean to measure the baryonic density of the Universe. See the review of Spite \& Spite (2010) on lithium and that of Steigman (2010) on nucleosynthesis in this volume, for an updated view of the problem. The most striking development came from the accurate measurement of the baryonic density from the WMAP satellite (Dunkley et al. 2009) which, coupled with standard BBN, implied a primordial Li abundance a factor of three to five higher than the Spite plateau. This discrepancy is often referred to as the "cosmological lithium problem".

Many solutions for this discrepancy have been proposed, including new physics at the time of the Big Bang (see for example Jedamzik 2004, 2006, Jittoh et al. 2008, Hisano et al. 2009), astration in the pristine Galaxy (Piau et al. 2006) and turbulent diffusion to deplete lithium in the stellar atmospheres (Richard et al. 2005). A fresh look at the problem can be afforded by the study of lithium in metal-poor populations of external galaxies. Theories like that of Piau et al. (2006) can be immediately tested and also theories which invoke stellar phenomena can be seriously constrained by the observation of stellar populations with different star formation histories. Unfortunately even nearby galaxies, like the Magellanic Clouds or the Sagittarius dwarf spheroidal are too far to allow such a study with existing telescopes, although they will be within the reach of the next generation of $40 \mathrm{~m}$ class telescopes.

There is, however, one external galaxy which is, just about, within reach of our telescopes: $\omega$ Cen. The complexity of its colour magnitude diagram clearly testifies the existence of multiple stellar populations with a range of metallicities. It is currently generally accepted that $\omega$ Cen is not a globular cluster, but a satellite galaxy of the Milky Way. It is more massive than all other globular clusters (about $2.5 \times 10^{5} \mathrm{M}_{\odot}$, Van de Ven et al. 2006), but was probably more massive in the past and has lost mass due to tidal interaction with the Galaxy. In a galaxy which has such a different mass and history with respect to the Milky Way, one should expect a very different lithium content of the metal-poor populations, if the "cosmological lithium problem" is due to astration by an early population. Also in the case of stellar phenomena, such as diffusion, one may expect different lithium content, if the stars show a consistent age spread. Finally we might be able to capture the results of Li production by super-AGB stars, with A(Li) up to 4 (Ventura \& D'Antona 2010, D'Antona \& Ventura 2010).

\section{Observations}

We have selected targets on the turn-off and sub-giant branch of $\omega$ Cen, mainly from the high precision FORS/VLT photometry of Sollima et al. (2005) and from the spectroscopic survey of Villanova et al. (2007). Ten targets were selected to trace the faint subgiant branch, called SGB-a in Sollima et al. (2005). Our targets are shown in Fig. 1, where we have used the wide field photometry of Bellini et al. (2009).

The targets were observed on three nights from April 27th to 29th 2007 at ESO Paranal with FLAMES at the Kueyen $8.2 \mathrm{~m}$ telescope. The fibres in Medusa mode fed the GIRAFFE spectrograph, configured in the HR15n setting, which covers both $\mathrm{H} \alpha$ and the LiI resonance doublet at $670.8 \mathrm{~nm}$ at a resolution of 17000 . The same plate configuration was observed for all the three nights, with integration times between one hour and slightly over two hours. Both plates were used alternatively and configured in such a way as to minimise the light loss due to atmospheric refraction. One further plate configuration was observed, with a partial overlap with the main plate configuration. We thus obtained a total integration time between 17 and 19 hours for 91 stars on the MS/SGB 


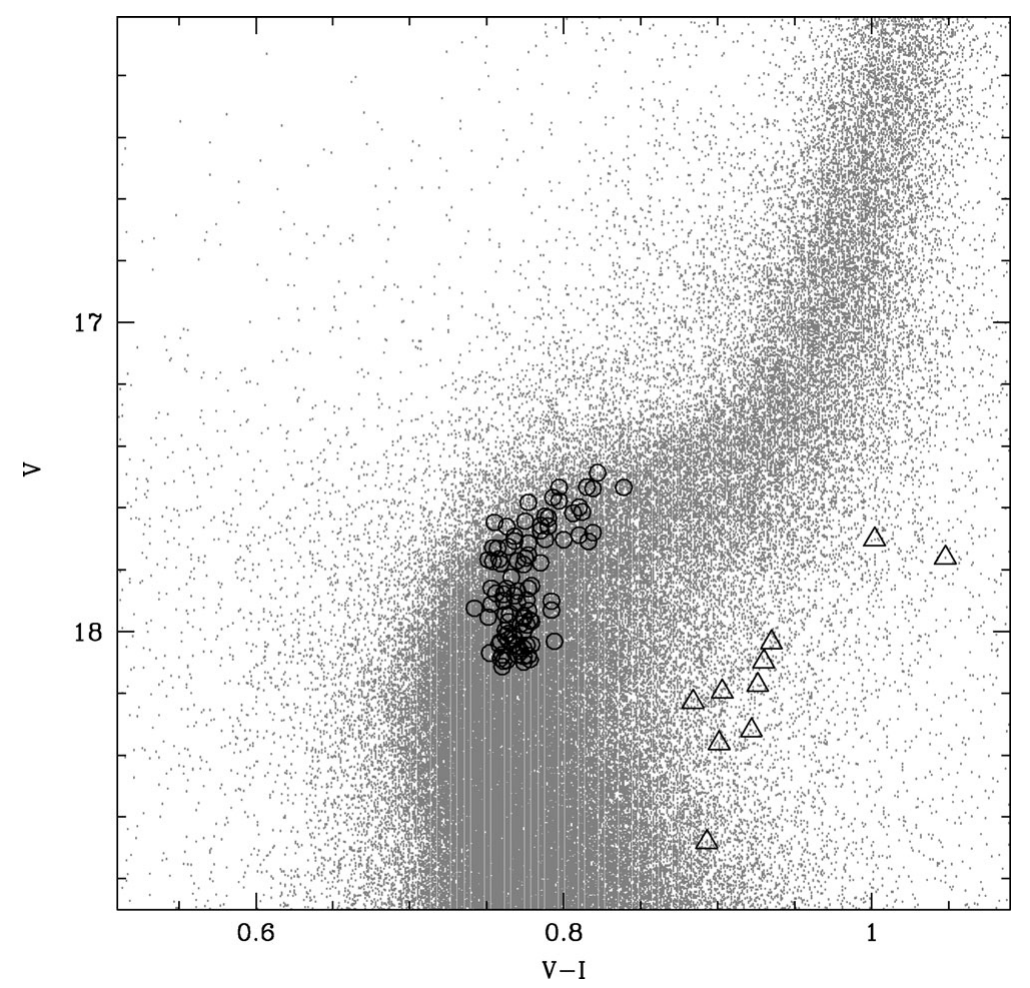

Figure 1. Colour-magnitude diagram of $\omega$ Cen from the WFI $/ 2.2 \mathrm{~m}$ photometry of Bellini et al. (2009) Our targets are shown as open circles, except for the targets on the SGB-a of Sollima et al. (2005), which are shown as open triangles.

and 10 stars on the SGB-a. After data reduction the spectra achieved $\mathrm{S} / \mathrm{N}$ ratios in the range 30 to 90 with a mean around 60 .

\section{Analysis}

Our analysis is based on one dimensional model atmospheres computed with version 9 of the ATLAS code (Kurucz 2005) in its Linux version (Sbordone 2005, Sbordone et al. 2004). The effective temperature of the stars has been determined by fitting the wings of $\mathrm{H} \alpha$. The theoretical profiles were computed with a modified version of the BALMER code $\nmid$ which uses the Barklem et al. $(2000 \mathrm{a}, \mathrm{b})$ self broadening theory and Stehlé \& King (1999) Stark broadening. At this stage we assumed $\log g=4.0$ and a metallicity of -1.5 for all stars, thus ignoring the dependence of the Balmer line profiles on metallicity and surface gravity. The equivalent widths of the Li I resonance doublet were measured by fitting synthetic profiles, as done in Bonifacio et al. (2002). When we could not detect the Li line we estimated an upper limit as $2 \sigma_{E W}$, where $\sigma_{E W}$ was estimated from the Cayrel formula (Cayrel 1988). A model atmosphere with the appropriate effective temperature, $\log \mathrm{g}=4.0$ and metallicity -1.5 was computed for each star and synthetic profiles were iteratively computed with SYNTHE until the equivalent width of the Li doublet matched the measured equivalent width. A microturbulent velocity of $1 \mathrm{kms}^{-1}$ was assumed, this, like the assumed surface gravity and metallicity, have effects of a few hundredths of

$\dagger$ The original version of R.L. Kurucz is available at http://kurucz.harvard.edu/ 


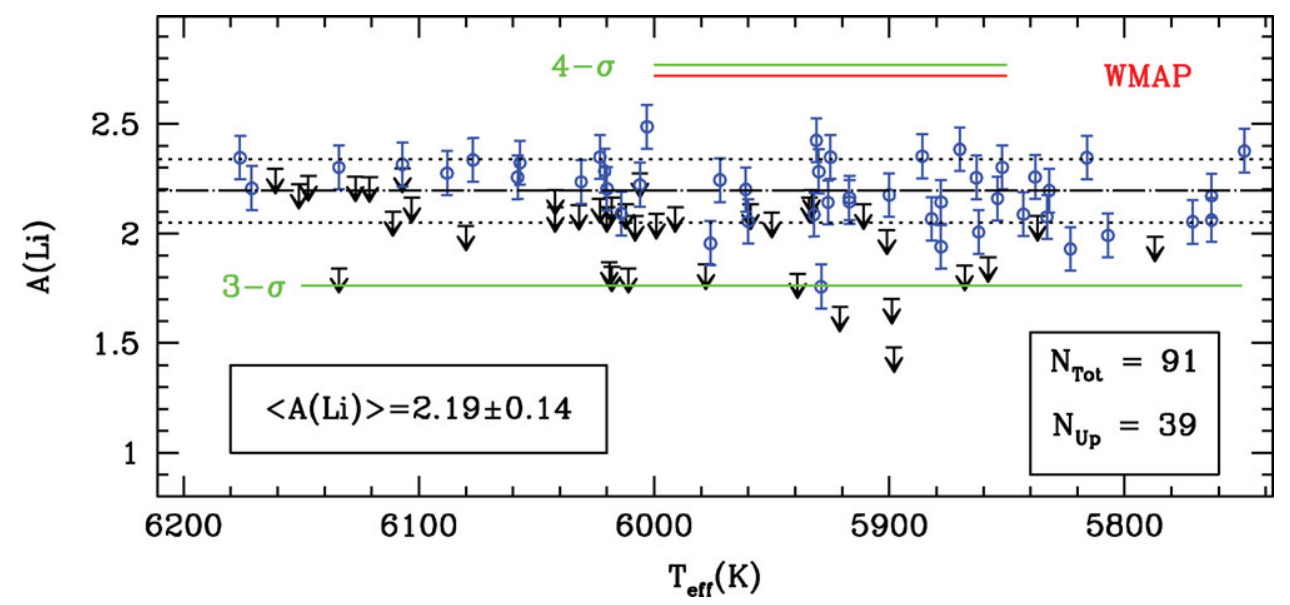

Figure 2. Li abundances in sub-giant and turn-off stars of $\omega$ Cen as a function of effective temperature.

dex on the derived $\mathrm{Li}$ abundance, this is totally negligible in the current context. Our $\mathrm{S} / \mathrm{N}$ ratios are high enough that the error on $\mathrm{Li}$ abundances is totally dominated by the uncertainty in effective temperatures. The latter is of the order of $150 \mathrm{~K}$ and is dominated by the uncertainty in the correction of the blaze function of GIRAFFE. Our estimated total uncertainty on the $\mathrm{Li}$ abundance is 0.1 dex.

\section{Results}

For none of the stars of the SGB-a did we detect the Li doublet. For the other stars our results are summarised in Fig. 2, out of a total of 91 MS/SGB stars for 39 we could not detect any lithium. For the stars with measured $\mathrm{Li}$ the abundance appears to be uniform without any trend with effective temperature, the mean value is $\mathrm{A}(\mathrm{Li})=2.19$ with a dispersion of 0.14 dex. We verified that if we use effective temperatures based on the $\mathrm{V}-\mathrm{I}$ colour the mean $\mathrm{Li}$ abundance is similar (2.21) and the dispersion is a little smaller (0.11).

The spectral region covered by our observations is not rich of metallic lines, nevertheless we used the Fe I and Ca I to derive the metallicity of the stars, assuming $[\mathrm{Ca} / \mathrm{Fe}]=+0.4$. For the stars in common we get a rather good agreement with the results of Villanova et al. (2007) although we note a small offset of the order of 0.1 dex. Although this is, perhaps, not surprising, given the different spectral range, resolution, model atmospheres and spectrum synthesis codes used, we consider these metallicities yet preliminary and we plan to further investigate these differences in the future. However, even with these preliminary metallicities it is clear that there are no obvious trends of $\mathrm{Li}$ abundance with metallicity. Also for the upper limits, there does not appear to be present any clustering of upper limits at a particular range of metallicities.

\section{Discussion}

It appears that the stars of $\omega$ Cen lie on the Spite plateau. Comparison with the Galactic field stars, on the same effective temperature scale (see Sbordone et al. 2010) 
shows that the stars of $\omega$ Cen occupy the same zone of the Galactic stars, both in the $\mathrm{A}(\mathrm{Li})-\mathrm{T}_{\text {eff }}$ and in the $\mathrm{A}(\mathrm{Li})-$ metallicity planes. This brings us to two robust conclusions:

- the Spite plateau exists also in other galaxies;

- the mechanism(s) which cause the "cosmological lithium problem" are the same in the Milky Way and in other galaxies.

Although this has only been established for $\omega$ Cen, it is simpler to assume that this is true for all galaxies than to assume that all galaxies behave differently and that only $\omega$ Cen behaves like the Milky Way. Until Li is measured in further external galaxies, this is an acceptable working hypothesis.

These facts immediately tell us that explanations of the "cosmological lithium problem" which require a special evolution for the Milky Way Halo are immediately ruled out. This is the case of the model by Piau et al. (2006), which requires that from one third to one half of the Galactic Halo $\left(\sim 10^{9} \mathrm{M}_{\odot}\right)$ has been processed through massive stars which effectively depleted lithium from the primordial value to the current observed Spite plateau. It would be extremely contrived to assume that another galaxy, of current mass $2.5 \times 10^{6} \mathrm{M}_{\odot}$, thus of very different type and evolution from the Milky Way, has undergone a similar process with a fine tuning of the mass fraction processed through massive stars so that the Spite plateau results identical to that of the Milky Way. Occam's razor requires that this theory be discarded.

Among other solutions of the "cosmological lithium problem" all which invoke stellar atmospheric phenomena, such as diffusion might also be tightly constrained by our observations. All such phenomena are time dependent and they may produce a uniform Spite plateau in the Galactic Halo, only because the age-spread in the Halo is very small. So the question is: what is the age spread among the stars observed by us in $\omega$ Cen? For the stars in common with Villanova et al. (2007) we may use their age estimates, based on theoretical isochrones, their metallicity estimates and the requirement that, in the colour-magnitude diagram, each sub-giant branch be associated to a Main Sequence which contains the same relative number of stars. Such ages are given in their table 2 and for the stars in common with our study we find the age spread is 5.6 Gyr. If the age spread in $\omega$ Cen is indeed so large, then all theories which invoke time-dependent phenomena, such as diffusion, would be ruled out, since it would be impossible for stars of such different ages, which have started their lives with the same (primordial) Li abundance, to still show the same Li abundance.

However the actual age spread in $\omega$ Cen is still a matter of debate and the relative ages of Villanova et al. (2007) stand out in the literature for providing the largest spread. Our sample of stars captures essentially the metal-poor population of $\omega$ Cen. According to the vast majority of studies, the intrinsic age spread of this population is consistent with zero, from the first photometric estimates (e.g., Hughes et al. 2004, Hilker et al. 2004), to the most recent spectro-photometric studies based on high precision HST CMDs and low resolution spectroscopy of vast samples of SGB and TO stars (such as Sollima et al. 2005, Stanford et al. 2006, Kayser et al. 2006). Theoretical work supports these findings, implying that a first, coeval generation of stars (the metal-poor population) is responsible for at least part of the pollution of the subsequent generations (see e.g., Norris 2004, Lee et al. 2005, Romano et al. 2009). The uncertainties of the experimental age spread determination procedures still allow to accommodate for a maximum spread - within the metal-population - of about 1 Gyr. Besides Villanova et al. (2007), also the spectroscopic study by Johnson et al. (2009), supports a consistent age spread. In this study the metal-poor stars show different degrees of s-process enrichment (see their Figure 13), implying some 0.1-3 Gyrs (Schaller et al. 1992) for intermediate-mass AGB 
stars to pollute part of the metal-poor group, depending on the actual mean mass of the AGB population.

It is thus clear that at the present state of understanding of the age spread in $\omega$ Cen our observations do not provide a strong constraint on the viability of diffusionlike mechanisms for Li depletion. Future spectroscopic and photometric observations are likely to better pinpoint this issue. It is possible that even a spread of 1 Gyr would prove a strong constraint on possible Li depletion mechanisms

\section{References}

Barklem, P. S., Piskunov, N., \& O'Mara, B. J. 2000a, A\& $A$ (Letters), 355, 5

Barklem, P. S., Piskunov, N., \& O'Mara, B. J. 2000b, A\&AA, 363, 1091

Bellini, A., Piotto, G., Bedin, L.R., Anderson, J., Platais, I. et al. 2009, A\&A, 493, 959

Bonifacio, P. et al. 2002, A\&GA, 390, 91

Cayrel, R. 1988, in The Impact of Very High S/N Spectroscopy on Stellar Physics, G. Cayrel de Strobel and M. Spite eds., IAU Symp. 132, p. 345

D'Antona, F. \& Ventura, P. 2010, this volume

Dunkley, J. et al. 2009, ApJS, 180, 306

Hilker, M., Kayser, A., Richtler, T., \& Willemsen, P. 2004, A\&A(Letters), 422, 9

Hisano, J., Kawasaki, M., Kohri, K., \& Nakayama, K. 2009, Phys. Rev. D, 79, 063514

Hughes, J., Wallerstein, G., van Leeuwen, F., \& Hilker, M. 2004, AJ, 127, 980

Jedamzik, K. 2004, Phys. Rev. D, 70, 083510

Jedamzik, K. 2006, Phys. Rev. D, 74, 103509

Jittoh, T. et al. 2008, Phys. Rev. D, 78, 055007

Johnson, C. I., Pilachowski, C. A., Michael Rich, R., \& Fulbright, J. P. 2009, ApJ, 698, 2048

Kayser, A., Hilker, M., Richtler, T., \& Willemsen, P. G. 2006, A\& $A$, 458, 777

Kurucz, R. L. 2005, Memorie della Società Astronomica Italiana Supplementi, 8, 14

Lee, Y.-W. et al. 2005, ApJ(Letters), 621, 57

Norris, J. E. 2004, ApJ(Letters), 612, 25

Piau, L. et al. 2006, ApJ, 653, 300

Richard, O., Michaud, G., \& Richer, J. 2005, ApJ, 619, 538

Romano, D., Tosi, M., Cignoni, M., Matteucci, F., Pancino, E., \& Bellazzini, M. 2009, MNRAS, 1604

Sbordone, L. 2005, Memorie della Societa Astronomica Italiana Supplementi, 8, 61

Sbordone, L., Bonifacio, P., Castelli, F., \& Kurucz, R. L. 2004, Memorie della Societá Astronomica Italiana Supplementi, 5, 93

Sbordone et al. 2010, A\&A submitted

Schaller, G., Schaerer, D., Meynet, G., \& Maeder, A. 1992, A\&AS, 96, 269

Sollima, A., Ferraro, F. R., Pancino, E., \& Bellazzini, M. 2005, MNRAS, 357, 265

Spite, M. \& Spite, F. 1982a, Nature, 297, 483

Spite, F. \& Spite, M. 1982b, A\&A, 115, 357

Spite, M. \& Spite F. 2010, IAU Symposium 268: "Light elements in the Universe", C. Charbonnel, M. Tosi, F. Primas \& C. Chiappini, eds., this volume

Steigman, G. 2010, IAU Symposium 268: "Light elements in the Universe", C. Charbonnel, M. Tosi, F. Primas \& C. Chiappini, eds., this volume

Stanford, L. M., Da Costa, G. S., Norris, J. E., \& Cannon, R. D. 2006, ApJ, 647, 1075

Stehle, R. \& King, A. R. 1999, MNRAS, 304, 698

van de Ven, G., van den Bosch, R. C. E., Verolme, E. K., \& de Zeeuw, P. T. 2006, A\&SA, 445, 513

Ventura, P. \& D'Antona, F. 2010, MNRAS, in press, arXiv:0912.4399

Villanova, S. et al. 2007, ApJ, 663, 296 